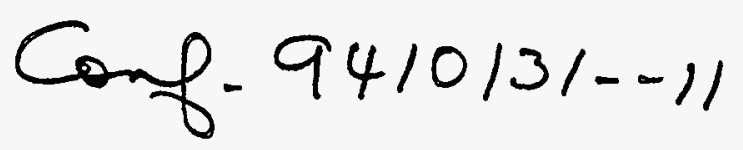

SAN094-1816C

\title{
AXIAL FOCUSING OF ENERGY FROM A HYPERVELOCITY IMPACT ON EARTH*
}

\author{
M. B. Boslough, E. P. Chael, T. G. Trucano and D. A. Crawford \\ Sandia National Laboratories, Albuquerque, NM 87185-0821

\begin{abstract}
Summary - We have performed computational simulations to determine how energy from a large hypervelocity impact on the Earth's surface would couple to its interior. Because of the first-order axial symmetry of both the impact energy source and the stresswave velocity structure of the Earth, a disproportionate amount of energy is dissipated along the axis defined by the impact point and its antipode (point opposite the impact). For a symmetric and homogeneous Earth model, all the impact energy that is radiated as seismic waves into the Earth at a given takeoff angle (ray parameter), independent of azimuthal direction, is refocused (minus attenuation) on the axis of symmetry, regardless of the number of reflections and refractions it has experienced. Material on or near the axis of symmetry experiences more strain cycles with much greater amplitude than elsewhere, and therefore experiences more irreversible heating. The focusing is most intense in the upper mantle, within the asthenosphere, where seismic energy is most effectively converted to heat. For a sufficiently energetic impact, this mechanism might generate enough local heating to create an isostatic instability leading to uplift, possibly resulting in rifting, volcanism, or other rearrangement of the interior dynamics of the planet. These simulations demonstrate how hypervelocity impact energy can be transported to the Earth's interior, supporting the possibility of a causal link between large impacts on Earth and major internally-driven geophysical processes.
\end{abstract}

\section{BACKGROUND}

Only since the advent of the space age has hypervelocity impact been recognized as an important process at work throughout the solar system. The appreciation of the degree of importance of the impact process continues to increase--underscored by this year's impact of comet Shoemaker-Levy 9 on Jupiter (e.g. Crawford et al., 1994)--and is probably not yet fully realized. The most obvious record of hypervelocity impacts is in the craters that they leave on the solid surfaces of planets and satellites; it was the cratered face of the moon that led to the first serious hypothesis invoking the process of hypervelocity impact by G.K. Gilbert (1893). Since then, understanding of the effects of hypervelocity impacts has focused on cratering (e.g. Melosh, 1988).

Hypervelocity impact is now known to have had many roles beyond making craters. For example, it is now recognized is that shock heating was a primary contributor to the thermal state of the Earth as it grew by accretion of material by impact (e.g. Kaula, 1979). A more recent development started with the discovery of a layer of iridium-enriched clay by Alvarez et al. (1980) and culminated with the identification and dating of the Chicxulub impact structure in Mexico (Hildebrand et al., 1991, Sharpton et al., 1992). Because of these discoveries, it is now widely accepted that a large hypervelocity impact was responsible for wiping out the dinosaurs and the majority of other life forms 65 million years ago, changing the natural history of life on Earth. In addition to that, a new consensus about the origin of the Moon has also developed over the last ten years. In the new theory, a monumental hypervelocity impact between the early Earth and another planet the size of Mars took place, melting most of both planets and ejecting the debris into orbit where it eventually became the Moon (e.g. Taylor, 1994). This same giant impact added enough

*This work was conducted at Sandia National Laboratories under the auspices of the Department of Energy under contract DE-AC04-94AL85000 under the Laboratory Directed Research and Development (LDRD) program. 


\section{DISCLAIMER}

Portions of this document may be illegible in electronic image products. Images are produced from the best available original document. 
angular momentum to increase the Earth's rotation rate in addition to keeping the moon in orbit. Computational simulations of this event (Kipp and Melosh, 1986) were instrumental in providing enough understanding of the physics of such giant impacts to allow this new theory to become accepted.

The cratering record has largely been erased on the Earth because of the constant destruction and renewal of its surface by plate tectonics, volcanic eruptions, glaciers, and weathering. However, comparison to the cratered lunar surface leads directly to the conclusion that the Earth has experienced hundreds of impacts leaving craters as big or bigger than Chicxulub, which by some estimates is $300 \mathrm{~km}$ in diameter. A reasonable question to ask is if there is other evidence of these impacts in the geologic record.

The purpose of our computational simulations is to begin to determine-the effect of hypervelocity impacts of this size on the internal workings of the Earth. Such perturbations may leave evidence that survives long after the impact structure on the surface has disappeared. If hypervelocity impacts can influence the Earth's interior, they may have played other roles in its history in addition to creating the Moon, determining the length of the day, and generating the conditions that allowed humans to evolve.

\section{HYPERVELOCITY IMPACTS AND GEOPHYSICAL PROCESSES}

There has been increasing speculation that energetic collisions have been responsible for processes as varied as continental flood basalt eruptions, mantle plumes, continental rifting, and geomagnetic pole reversals. The link between impacts and such geophysical processes was first discussed by Seyfert and Sirkin (1979), who suggested that impact-induced mantle plumes could be a mechanism for initiating the breakup of plates. Burek and Wanke (1988) listed correlations between known Cenozoic impacts and geomagnetic field reversals, unconformity ages, shifts in paleotemperatures, and tectonic episodes. They suggested that major impacts could generate shock-induced phase transitions in the upper mantle and disrupt a delicately-balanced stability down to the core-mantle boundary. Rampino and Stothers (1988) proposed a quasi-periodic correlation between mass extinctions and major continental flood basalt volcanism over the last 250 million years and attempted to explain it in terms of episodic showers of impacting comets. Connections between impacts and the internal workings of the Earth are supported by correlations of the ages

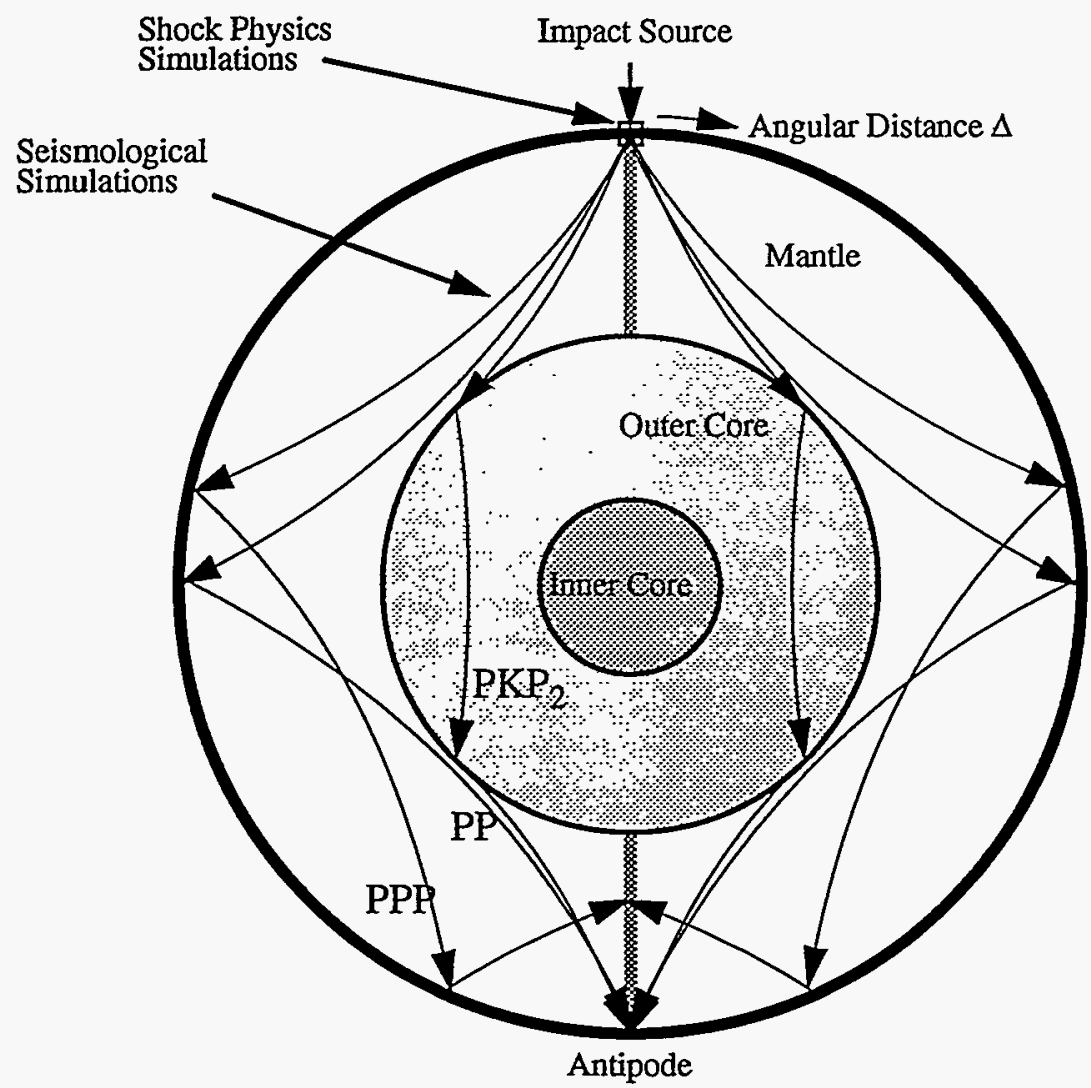

Fig. 1. Interior cross-section of the Earth illustrating the two computational domains. The shock physics simulations are limited to the impact source region, and the seismological simulations are over the entire interior. Various families of compressional seismic rays are drawn, showing how they focus on the surface at the impact antipode, or on the axis of symmetry. 
of tektites from strewn fields with geomagnetic field reversals (Glass, 1979), and by a reversal associated with sediments deposited immediately after the impact that formed the Ries Crater (Pohl, 1977).

A causal link between major impact events and global processes would probably require a significant change in the thermal state of the Earth's interior, presumably brought about by coupling of impact energy. One possible mechanism for such energy coupling from the surface to the deep interior would be through focusing due to axial symmetry (Figure 1). Antipodal focusing of surface and body waves from Earthquakes is a well-known phenomenon (Gutenberg and Richter 1934) which has previously been exploited by seismologists in studies of the Earth's deep interior (Rial, 1979; Chael, 1983). Antipodal focusing from impacts on the Moon, Mercury, and icy satellites has also been invoked by planetary scientists to explain unusual surface features opposite some of the large impact structures on these bodies (Schultz and Gault, 1975; Watts et al., 1991). For example, "disrupted" terrains have been observed antipodal to the Caloris impact basin on Mercury and the Imbrium basin on the Moon (Melosh, 1989). Very recently there have been speculations that antipodal focusing of impact energy within the mantle may lead to flood basalt and hotspot activity (Hagstrum and Turrin, 1991; Rampino and Caldeira, 1992) but there has not yet been an attempt at defining a mechanism and subjecting it to rigorous modeling.

The computational simulations presented in this paper are proof-of-principle tests for the coupling of energy from major impacts to the mantle by axial focusing of seismic waves. The situation is very different than for a giant earthquake which, in addition to having less energy, has an asymmetric focal mechanism and a larger source area. It should be noted that our hypothesis is fundamentally different than those proposed by many others (e.g. Green, 1972; Alt et al., 1988; Oberbeck et al., 1992; Negi, et al., 1993) which involve melting and excavation at the impact location. Problems with models of this type have been pointed out by Melosh (1989) and Loper (1991).

We are using two different types of simulations to model the asteroid impact and resulting seismic disturbance in the Earth's interior (Figure 1). A single computational method cannot accurately be applied to this problem because of the wide range in spatial and temporal scales and in peak stresses experienced. For example, during the impact event, the physical processes are occurring at scales on the order of the 10$\mathrm{km}$ diameter asteroid, and the peak shock pressures reach $6 \mathrm{Mbar}$. Much later, when the energy is being dissipated within the Earth, the peak stresses are in the 10 bar range, and the wave motion takes place within the $12,000-\mathrm{km}$ diameter of the Earth. Since it is computationally difficult to represent both extremes simultaniously, we have divided the problem into two parts. In the first part, we investigate the source region, where the stresses and strain rates are high, using CTH, the strong-shock hydrodynamics code. The second part makes use of a normal-mode summing seismological simulation that yields synthetic seismograms for various locations within the Earth. These simulations demonstrate that displacement and strain amplitudes at the surface of the Earth near the antipode are orders of magnitude larger than those over most of the rest of the Earth's surface, and that the seismic energy remains sharply focused down to the core-mantle boundary.

\section{SHOCK PHYSICS SIMULATIONS}

We have investigated the source region of a 10-km diameter asteroid impacting at $20 \mathrm{~km} / \mathrm{s}$ using $\mathrm{CTH}$, the multi-dimensional multi-material elastic-plastic Eulerian code developed at Sandia National Laboratories (McGlaun et al., 1990). This work is in progress, and to date we have completed two-dimensional axisymmetric simulations to determine the influence of asteroid shape upon its interaction with the Earth in the near field (to a depth of $50 \mathrm{~km}$ below the impact point). The Earth is modeled using an ANEOS equation of state (Thompson, 1989), which contains thermodynamically consistent solid/melt and liquid/vapor phase transitions. Within a few seconds after impact, major shape differences become difficult to distinguish (Figure 2), demonstrating that, to first order, such an impact can be simulated as a point source when modeling far-field effects of the impact.

The results of these near-field shock physics simulations can be used to generate source functions for the seismological simulations that are more accurate than the point source approximations, which will in turn yield more accurate strain histories. Shock physics simulations will be performed in a stratified, gravitationally-stable Earth model. Because the seismological simulations assume linear elastic wave propagation, the shock physics simulations must be run until the maximum pressure at the shock front drops below the elastic limit. We expect that the deviations from point source functions will become more important as larger impacts are investigated. We will also use 3-D computational simulations to investigate the effects of impact angle. 

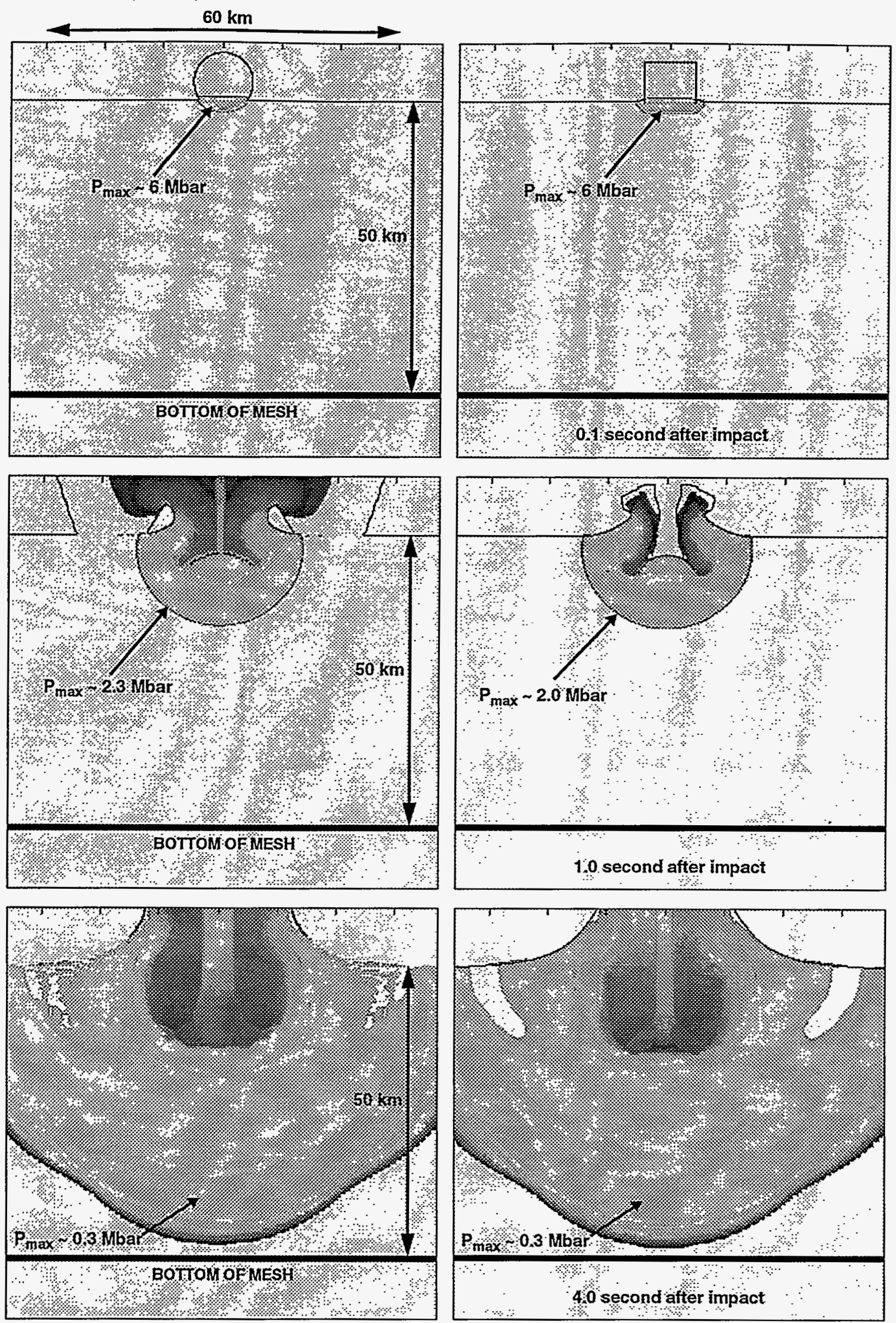

Figure 2. CTH simulations of the source region of the impact of a $10-\mathrm{km}$ diameter asteroid at $20 \mathrm{~km} / \mathrm{s}$ onto the Earth, demonstrating that shape has little effect after a few seconds and a point source function for seismological simulations is a good initial approximation. 
As suggested by the results of the previous section, seismological simulations were carried out to predict the global response to a hypervelocity impact by using a point source function. Displacement, stress and strain time histories were modeled through the use of normal mode synthetics by adding Earth's vibrational modes in the frequency domain (Chael, 1983). Each of the Earth's normal modes contributes a decaying sinusoid to the particle motion at a point in the Earth. The contribution of an individual mode to the observed motion at some point is a complex function of the source mechanism and the locations of the source and observation point. For our synthetics, we used the elastic, spherically-symmetric Earth model 1066A of Gilbert and Dziewonski (1975), for which there are existing compilations of the eigenfrequencies and radial eigenfunctions. The attenuation profile of the PREM model (Preliminary Reference Earth Model, Dziewonski and Anderson, 1981) was used to calculate the $Q$ value (a measure of attenuation) for each of the $1066 \mathrm{~A}$ modes.

The impact source was modeled as a vertical point force applied at the Earth's surface as a delta function in time. Assuming the impactor had a diameter of $10 \mathrm{~km}$ and an average density of $3 \mathrm{gm} / \mathrm{cm}^{3}$, and that it collided with Earth at $20 \mathrm{~km} / \mathrm{s}$, we estimated the source impulse to be $3 \times 10^{24}$ dyne-sec. Because of the symmetry, the Earth's toroidal modes are not excited by a vertical point force, thus only spheroidal modes were included in the calculations. Our synthetics represent the sum of 3382 modes, all of 1066A's spheroidal modes with frequencies less than $0.022 \mathrm{~Hz}$ (or periods greater than 45 seconds).
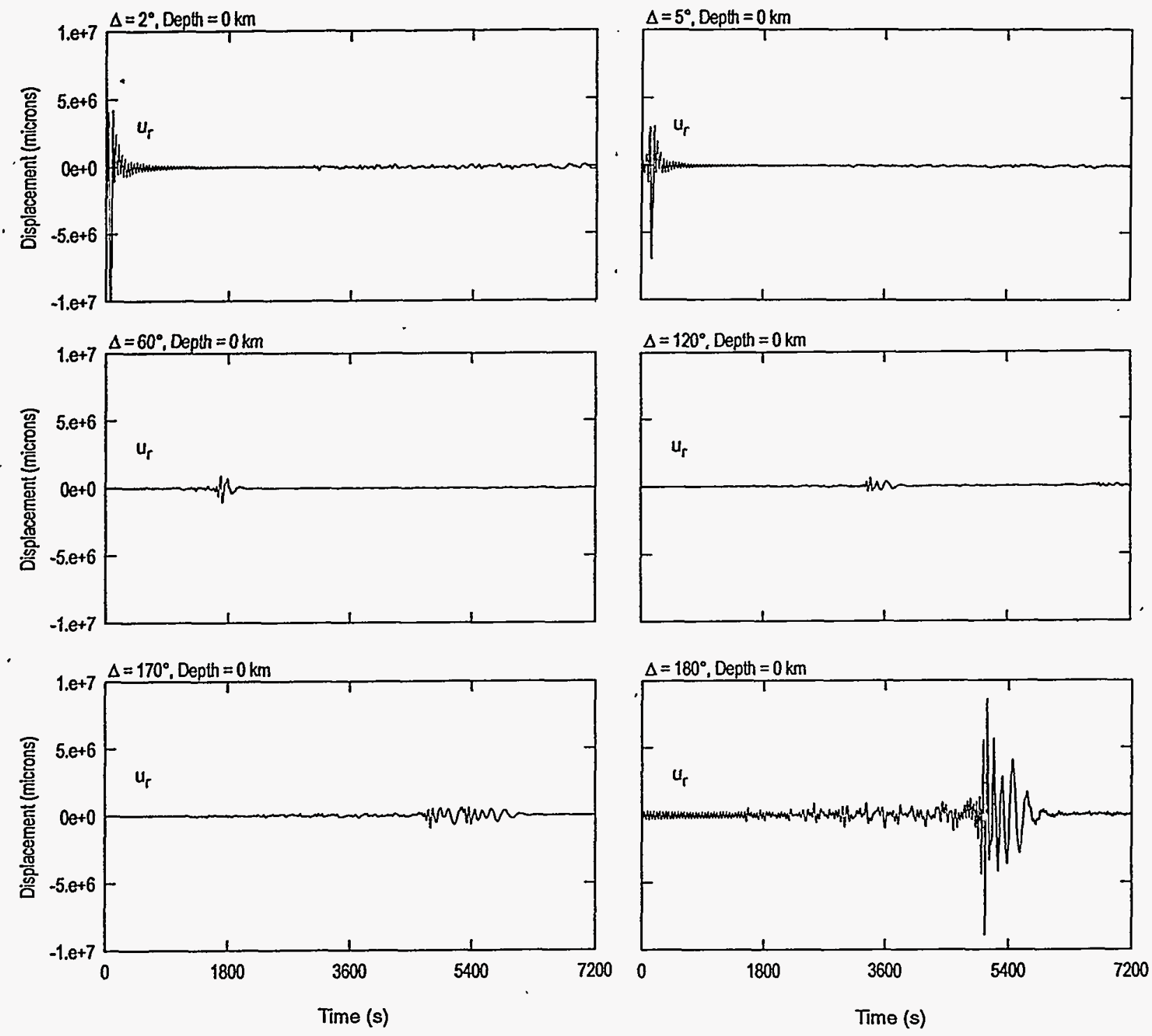

Figure 3. Radial displacement histories on the Earth's surface at six different angular distances from the impact. The peak amplitude at the antipode approaches 10 meters, similar to what it is only $2^{\circ}$ from the source. 
We generated synthetic displacement, stress and strain signals at several locations in and on the Earth, and measured the peak amplitudes from each of the signals. Figure 3 compares the displacement histories at six different angular distances from the source. This figure demonstrates the effect of antipodal focusing. Moving away from the source, the amplitudes of the signals decrease. Approaching the antipode (angular distance $=\Delta=180^{\circ}$ ), however, this trend reverses and there is a dramatic increase in amplitude as energy traveling along all azimuths from the source converges. Surface displacements at the antipode are more than an order of magnitude larger than over most of the Earth's surface. Figure 4 shows the peak strain registered on the surface as a function of distance from the source. In this figure one can see how sharply the energy is focused at the antipode. Note that the strain at the antipode reaches a level comparable to that only a few degrees away from the impact.

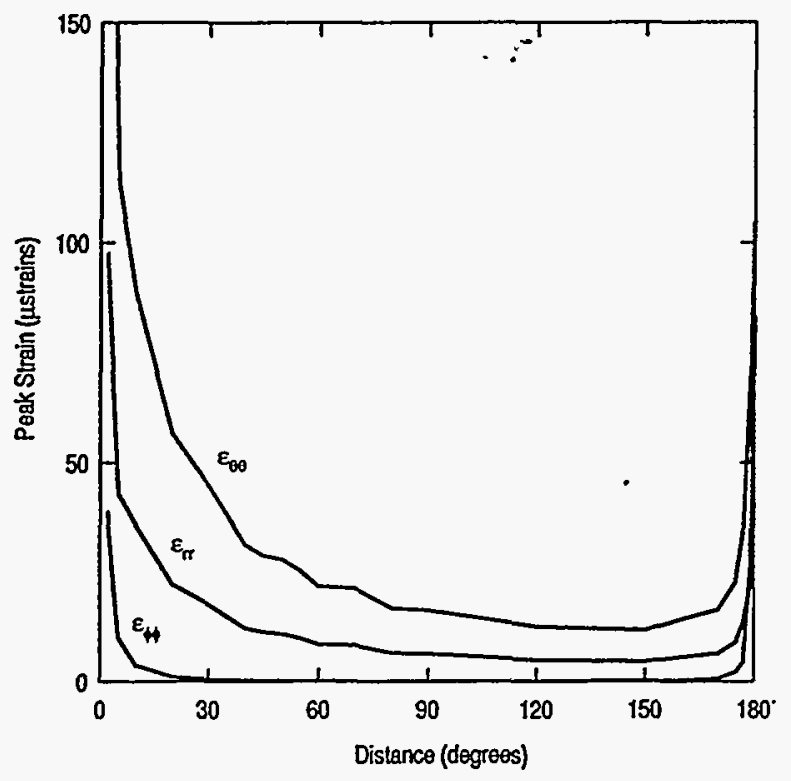

Fig. 4. Values of peak strain for three components, as a function of radial distance from the impact source.

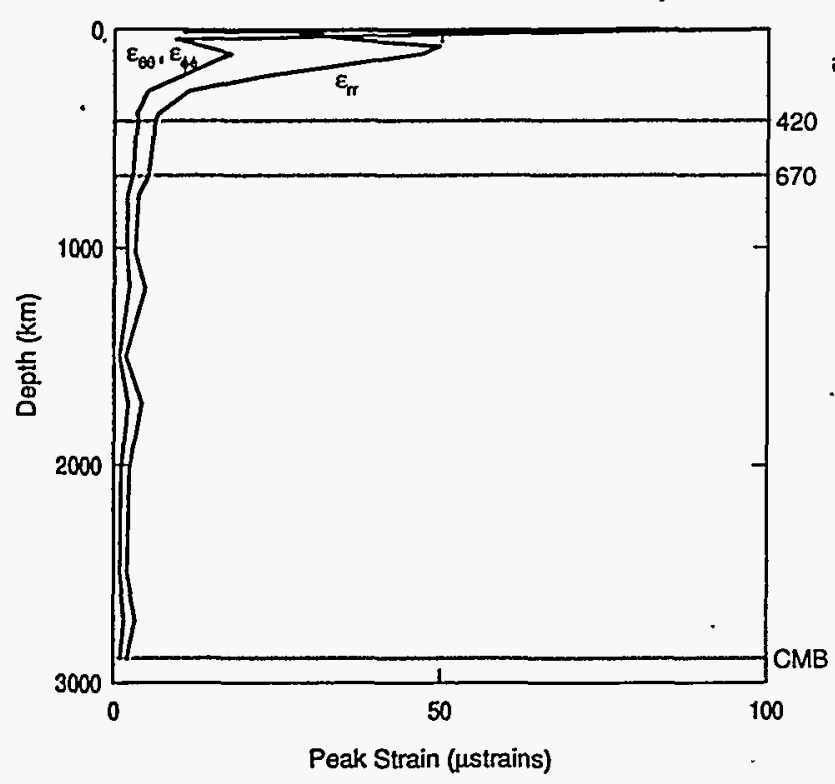

Fig. 6. Peak strains as a function of depth along the radius beneath the antipode down to the core-mantle boundary (CMB). Focusing is strongest within the asthenosphere, from about 100 to $300 \mathrm{~km}$ deep.

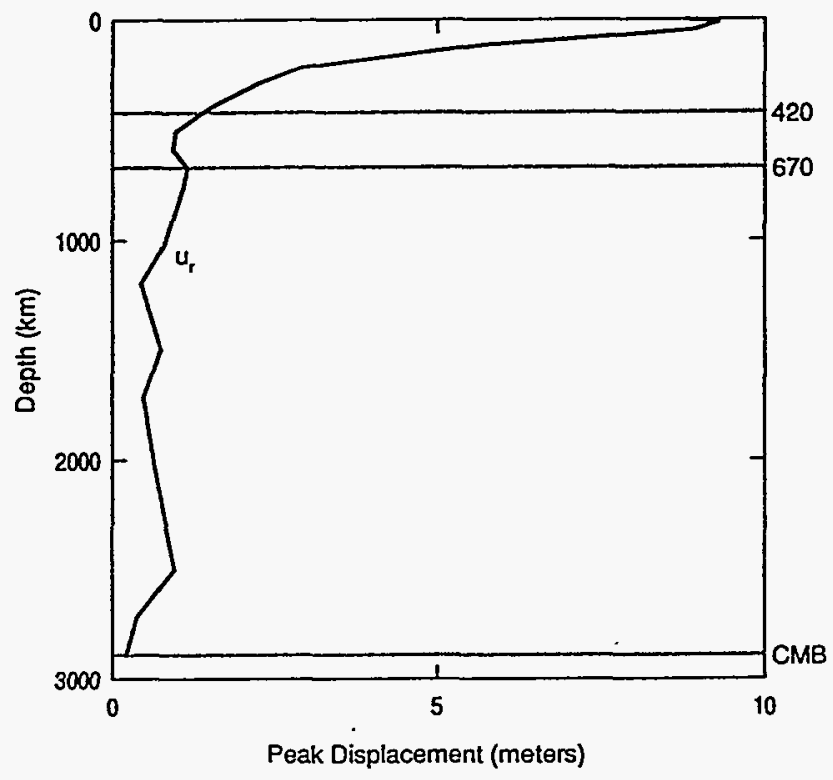

Fig. 5. Peak radial displacement as a function of depth along the radius beneath the antipode down to the core-mantle boundary (CMB).

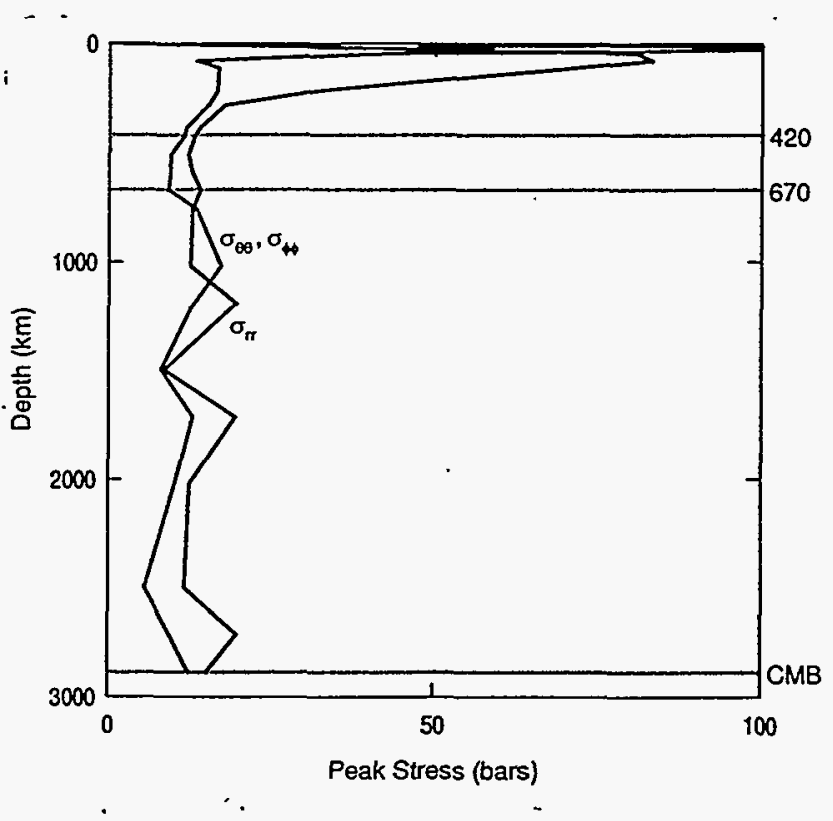

Fig. 7. Peak stresses as a function of depth along the sub-antipodal axis down to the core-mantle boundary (CMB). 
Figure 5 shows the variation of peak displacement amplitude with depth beneath the antipode, from the surface to the core-mantle boundary (CMB). Similarly, Figures 6 and 7 present the variation of peak strain and peak stress, respectively, with depth. Figures 5-7 demonstrate that the largest motions along the antipodal axis occur in the upper $200 \mathrm{~km}$. These large amplitudes are due to the fundamental-mode Rayleigh surface waves. At greater depths the Rayleigh-wave contribution diminishes, and the body arrivals become relatively more prominent. The fact that strain amplitudes are high in the asthenosphere (depths between about 100 to $300 \mathrm{~km}$ ) is an important result, because 1) this is the region where seismic waves are most efficiently attenuated, and 2) basaltic eruptions can result from melting of this part of the mantle

Though the peak amplitudes decrease substantially with depth beneath the antipode, the signals at any depth still represent focused arrivals, with amplitudes much larger than seen at similar depths away from the antipodal axis. Figures 8-10 show the displacement and strain records for three different locations on the core-mantle boundary. Figure 8 displays the motions on the CMB directly beneath the impact. The direct arrival, with a spherically spreading wavefront, is notably weaker than later focused arrivals. At an angular distance of $90^{\circ}$ on the CMB (Figure 9), there is no focusing and the amplitudes remain small throughout the signal. Figure 10 shows the motions on the CMB beneath the impact's antipode. Comparing Figures 8 and 10, one sees that the motions on the CMB are actually greater beneath the antipode than beneath the source location.

To improve on the seismic modeling, we plan to expand the set of included modes in order to extend the synthetics to higher frequencies. The body wave arrivals in particular will be better modeled by using a larger bandwidth. The shock physics simulations will be used to constrain the input source model. Finally, we will estimate the degradation in the focusing on the antipodal axis due to the Earth's asymmetry and lateral heterogeneity.
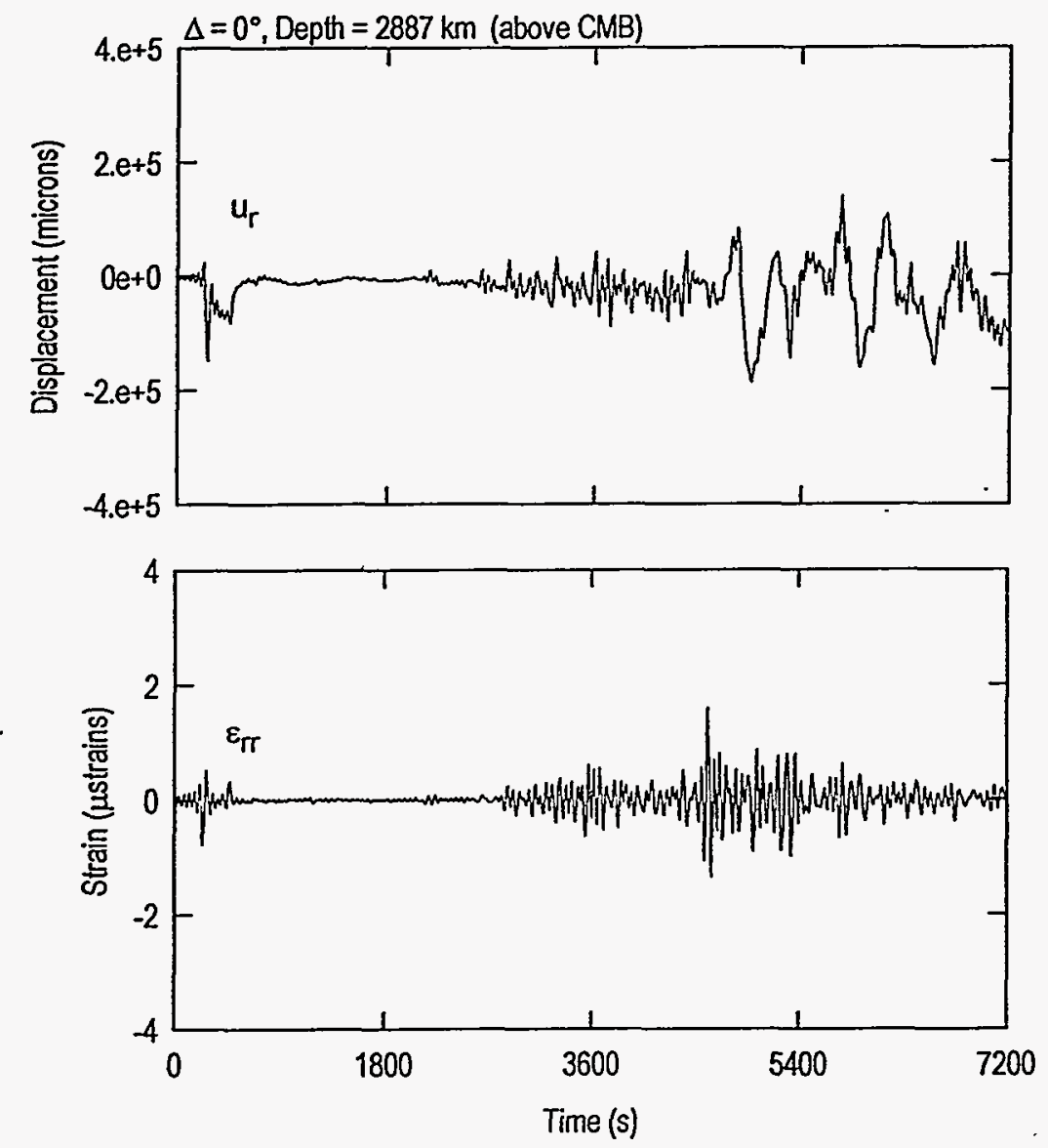

Fig. 8. Radial displacement and strain histories just above the core-mantle boundary (CMB) directly beneath the impact source. 

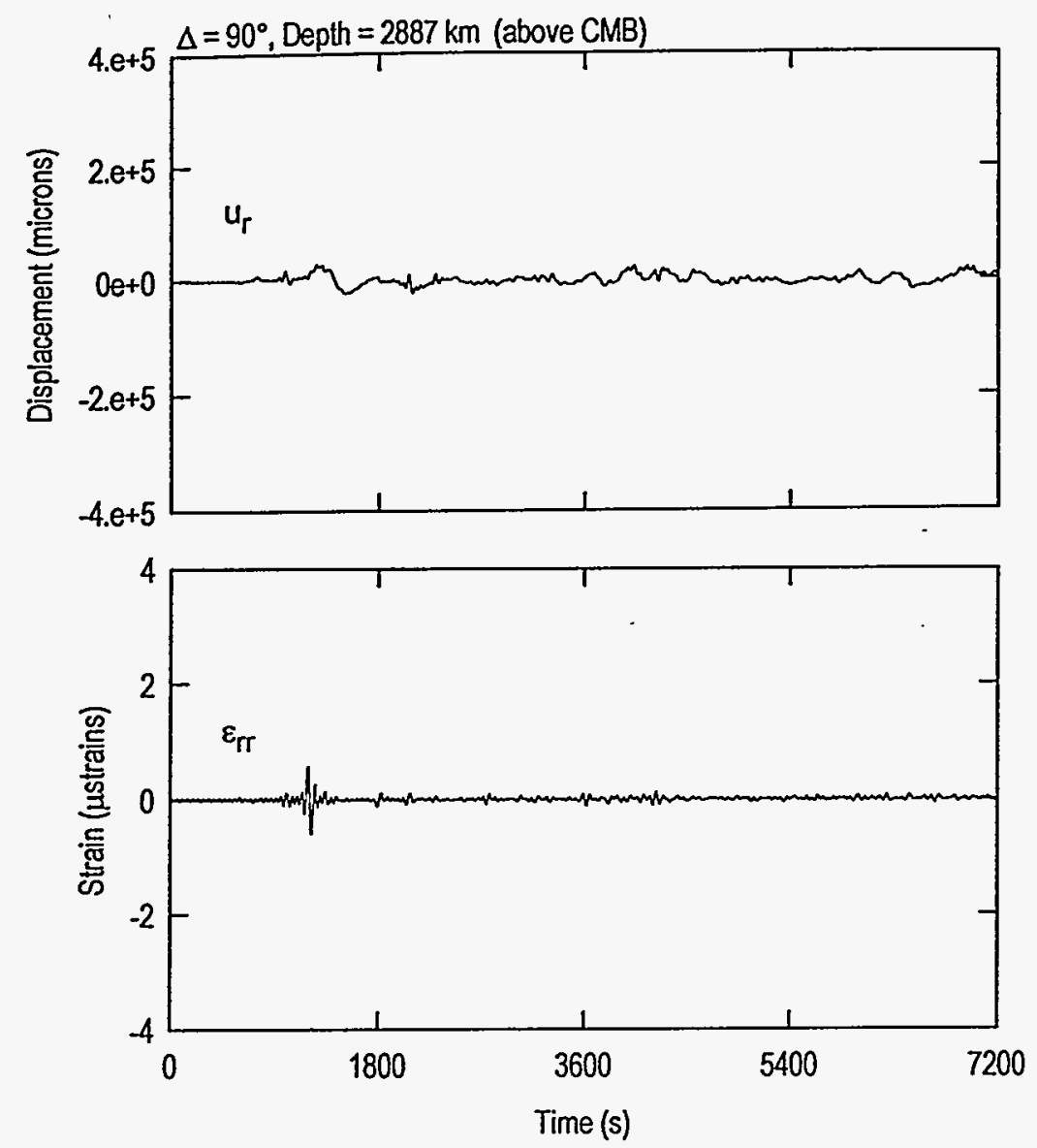

Fig. 9. Radial displacement and strain histories just above the CMB between the impact and antipode.

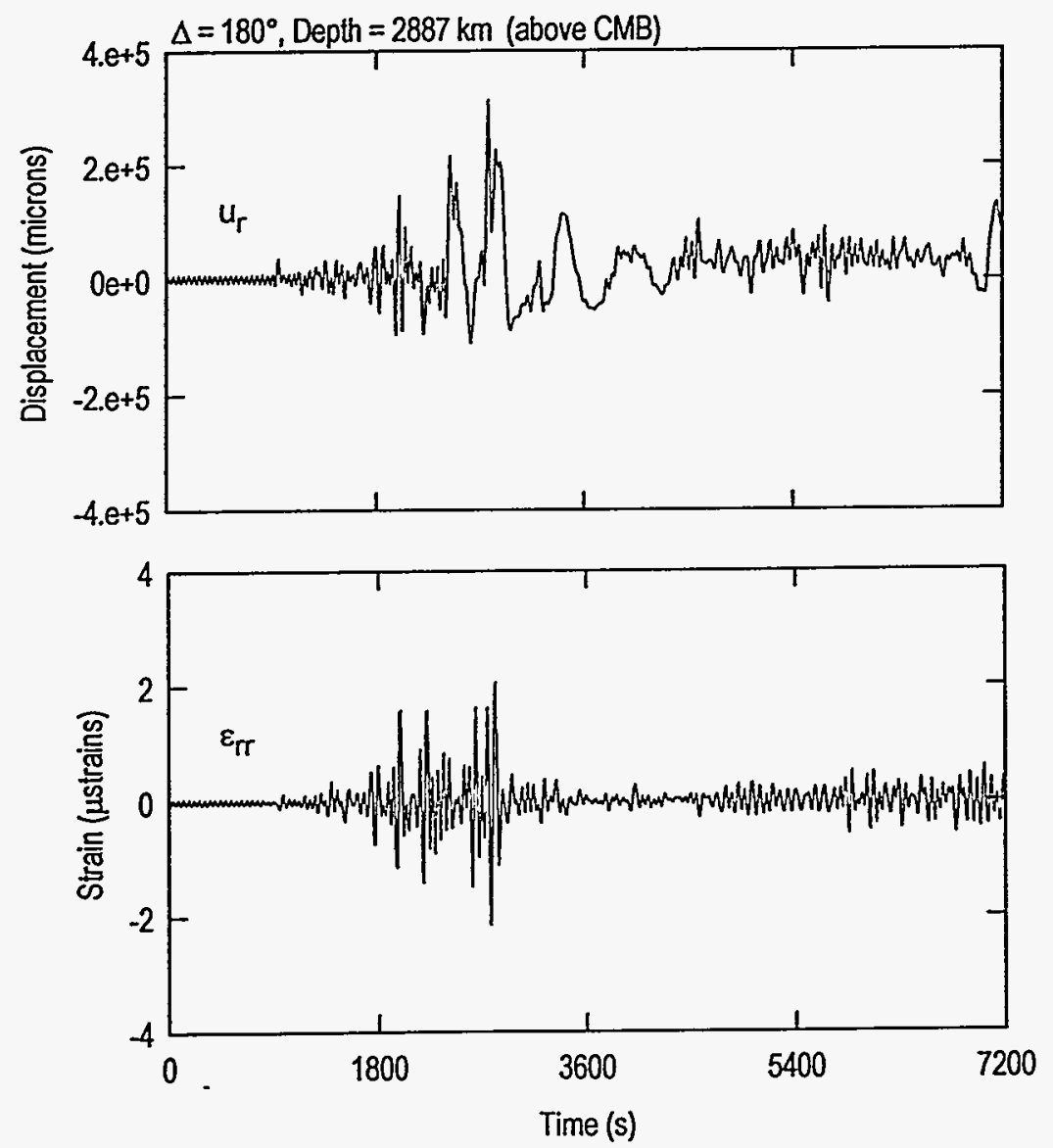

Fig. 10. Radial displacement and strain histories just above the CMB directly beneath the antipode. 


\section{CONCLUSIONS}

Our simulations show that displacement and strain amplitudes at the surface near the antipode (angular distance $\Delta=180^{\circ}$ ) of a large impact event on the Earth are orders of magnitude larger than those over most of the rest of the Earth's surface. For an impact of the size that occurred 65 million years ago, the peak displacement at the antipode approached ten meters, exceeding the ground motion only a few hundred kilometers from the source. Seismic energy is most strongly focused in the upper mantle, within the asthenosphere, where is is most readily converted to heat. Peak strains fall off rapidly with depth, but remain sharply focused along the impact-antipodal axis down to the core-mantle boundary. Future work will couple the shock physics simulations of the source region directly to the seismological model and include simulations of oblique impacts and larger impacts. We will also apply a dissipation model to determine the extent of the resulting thermal anomaly in the Earth's interior.

\section{ACKNOWLEDGMENTS}

This work was supported by the United States Department of Energy under Contract DE-AC04$94 A L 85000$ and was funded by the LDRD program.

\section{REFERENCES}

Alt D., J.M. Sears, and D. W. Hyndman (1988). Terrestrial Maria: the Origins of Large Basalt Plateaus, Hotspot Tracks and Spreading Ridges, J. Geol., 96, 647-662.

Alvarez, L.W., W. Alvarez, F. Asaro and H.V. Michel (1980). Extraterrestrial Cause of the Cretaceous/Tertiary Extinction, Science, 208, 1095-1108.

Burek P.J. and H. Wanke (1988). Impacts and glacio-eustay, Plate-Tectonic Episodes, Geomagnetic Reversals: a Concept to Facilitate Detection of Impact Events. Phys. Earth Planet. Inter., 50, 183-194.

Chael (1983) Constraints on the Earth's Anelastic and Aspherical Structure from Antipodal Surface Waves, Ph.D. Thesis, Caltech.

Crawford, D.A., M.B. Boslough, T.G. Trucano and A.C. Robinson (1994). The Impact of Periodic Comet Shoemaker-Levy 9 on Jupiter, Int. J. Impact. Engng., this volume.

Dziewonski A.M. and D.L. Anderson (1981) Preliminary Reference Earth Model, Phys. Earth Planet. Inter., 25, 297-356

Gilbert, G.K. (1893) The Moon's Face: A Study of the Origin of its Features, Bull. Phil. Soc. Wash., 12, 241-292.

Gilbert F. and A.M. Dziewonski (1975) An Application of Normal Mode Theory to the Retrieval of Structural Parameters and Source Mechanisms from Seismic Spectra, Phil. Trans. Roy. Soc. Lond. A, 278, 187-269

Glass B.P. et al. (1979) Proc. Lunar Planet. Sci. Conf. 10th, pp. 25-37, NY: Pergamon.

Green D.H. (1972) Archaen Greenstone Belts May Include Terrestrial Equivalents of Lunar Maria? Earth and Planet. Sci. Lett., 15, 263-270.

Gutenberg B. and Richter C. (1934) Gerlands Beitr. Geophys., 43, 56.

Hagstrum J.T. and Turrin B.D. (1991) Is Flood Basalt Volcanism a Seismically-Induced Response to Large Antipodal Bolide Impacts? EOS, 72 (44), p. 516.

Hildebrand, A.R., G.T. Penfield, D.A. Kring, M. Pilkington, Z.A. Carmargo, S.B. Jacobson, and W.V. Boynton (1991) Chicxulub Crater: A Possible Cretaceous/Tertiary Boundary Impact Crater on the Yucatan Peninsula, Mexico, Geology, 19, 193-206.

Kaula, W.M. (1979). Thermal Evolution of Earth and Moon Growing by Planetesimal Impacts, J. Geophys. Res., 84: 999-1008.

Kipp, M.E. and H.J. Melosh (1986). Origin of the Moon: A Preliminary Numerical Study of Colliding Planets, Lunar and Planetary Science XVII, 420-421.

Loper D.E. (1991) Mantle Plumes, Tectonophys., 187, 373-384.

McGlaun, J. M., S. L. Thompson, and M. G. Elrick (1990). CTH: A Three Dimensional Shock-Wave Physics Code, Int. J.; Impact. Engng., 10, 351.

Melosh, H.J. (1989). Impact Cratering: A Geologic Process, Oxford U. Press, New York.

Negi J.G., P.K. Agrawal, O.P. Pandey and A.P. Singh (1993). A possible K-T Boundary Bolide Impact Site Offshore Near Bombay and Triggering of Rapid Deccan Volcanism, Phys. Earth Planet. Int., 76, 189197. 
Oberbeck V.R., J.R. Marshall and H. Aggarwal, (1992). Impacts, Tillites, and the Breakup of Gondwanaland, J. Geol., 101, 1-19.

Pohl, J. (1977). Paleomagnetische und Gesteinsmagnetische Untersuchungen an den Kermen der Forschungsbohrung Noerdingen, Geol. Bavarica, 75, 329-348.

Rampino M.R. and Stothers, R.B. (1988). Flood Basalt Volcanism During the Past 250 Million Years, Science, 241, 663-668.

Rampino M.R. and Caldeira K. (1992). Antipodal Hotspot Pairs on the Earth, Geophys. Res. Lett., 19, 2011-2014.

Rial J.A. (1979) Seismic Waves at the Epicenter's Antipode, Part II, Ph.D. Thesis, Caltech

Schultz P.H. and Gault D.E. (1975). Seismic Effects from Major Basin Formation of the Moon and Mercury, The Moon, 12, 159-177.

Seyfert C.K. and Sirkin L.A. (1979) Earth History and Plate Tectonics. NY: Harper \& Row.

Sharpton, V.L, G.B. Dalrymple, L.E. Marin, G. Ryder, B.C. Schuraytz, and J.Urrutia-Fucugouchi (1992). New Links Between the Chicxulub Impact Structure and the Cretaceous/Tertiary Boundary, Nature, 359, 819-821.

Taylor. G.J. (1994). The Scientific Legacy of Apollo, Scientific American, July 1994, 40-47.

Thompson, S. L. (1989). ANEOS analytic equations of state for shock physics codes input manual, Sandia National Laboratories, SAND89-2951.

Watts A.W., R. Greeley and H.J. Melosh (1991) The Formation of Terrains Antipodal to Major Impacts, Icarus, 93, 159-168.

\section{DISCLAIMER}

This report was prepared as an account of work sponsored by an agency of the United States Government. Neither the United States Government nor any agency thereof, nor any of their employees, makes any warranty, express or implied, or assumes any legal liability or responsibility for the accuracy, completeness, or usefulness of any information, apparatus, product, or process disclosed, or represents that its use would not infringe privately owned rights. Reference herein to any specific commercial product, process, or service by trade name, trademark, manufacturer, or otherwise does not necessarily constitute or imply its endorsement, recommendation, or favoring by the United States Government or any agency thereof. The views and opinions of authors expressed herein do not necessarily state or reflect those of the United States Government or any agency thereof. 